\title{
Percepción del profesorado acerca de la importancia del uso de las tic para favorecer la inclusión del alumnado
}

\section{Teacher's perception of the importance of the use of ICTs to promote student inclusión}

DOI: $10.46932 / \mathrm{sfjdv} 2 \mathrm{n} 2-089$

Received in: March 1st, 2021

Accepted in: May 30th, 2021

\author{
Patricia Ortiz Marín \\ Graduada Educación Primaria. Especialista en Pedagogía Terapéutica. \\ Institution: RG Formación \\ Calle Rosalía de Castro, 44, 30107 Murcia \\ Ana Belén Giménez Velázquez \\ Graduada Educación Primaria. Especialista en Pedagogía Terapéutica. \\ Institution: RG Formación \\ Calle Rosalía de Castro, 44, 30107 Murcia \\ Jose María Rabal Alonso \\ Profesor ISEN Centro Universitario \\ Institution: ISEN \\ Calle Menéndez y Pelayo, 8, 30204 Cartagena, Murcia \\ E-mail: josemaria.rabal@um.es
}

\begin{abstract}
RESUMEN
Hoy en día, la educación inclusiva debería de ser un requisito en las aulas y aunque con el tiempo ha aumentado su presencia aún no está presente en todos los centros. Para que todos los discentes tengan igualdad de oportunidades y una educación de calidad se propone la incorporación de las TIC en las aulas. A través de una revisión bibliográfica se observan los beneficios que se pueden obtener a través de ellas, cuál es su uso y cómo implementarlas.

El siguiente trabajo tiene por objetivo ofrecer, a partir de un estudio detallado y la realización de una encuesta, la opinión de docentes sobre el uso de las TIC en las aulas con alumnos con NEE. Para llevar a cabo este trabajo, se ha realizado la encuesta a 81 docentes en activo de manera totalmente aleatoria para que los resultados sean lo más reales posibles.

Los resultados obtenidos muestran cómo la gran mayoría de los profesionales en educación están a favor de su inclusión en las aulas aunque para ello creen que deberían de recibir una formación adaptada a dichos contenidos.
\end{abstract}

Palabras clave: inclusión, CDPD, organización educativa, didáctica, equidad.

\footnotetext{
ABSTRACT

Nowadays, inclusive education should be a requirement in classrooms and although over time its presence has increased, it is still not present in all centers. In order for all students to have equal opportunities and a quality education, the incorporation of ICT in the classroom is proposed. Through a bibliographic review, the benefits that can be obtained through them, what is their use and how to implement them are observed.

The following work aims to offer, through a detailed study and a survey, the opinion of teachers on the use of ICT in classrooms with students with SEN. In order to carry out this work, the survey has been made to 81 active teachers in a completely random way so that the results are as real as possible.
} 
The results obtained show that the vast majority of education professionals are in favor of their inclusion in the classroom, although they believe that they should receive training adapted to these contents.

Key words: inclusion, CRPD, educational organization, didactics, equity.

\section{MARCO TEÓRICO}

Blanco (2008) nos dice que la educación de calidad implica un doble equilibrio entre el ofrecimiento de una educación común para todo tipo de alumnado, junto al reconocimiento y ajuste de las necesidades de aprendizaje y características individuales de los discentes. Y la excelencia y la equidad, siempre y cuando se hable del término calidad en general y no cuando solo unos pocos alumnos aprenden lo necesario para ser futuros participantes de nuestra sociedad.

Donde la igualdad de derechos y oportunidades en la educación necesita de un compromiso general de todos los agentes que conforman el sistema educativo (Escudero y Dominguez, 2012), demandando una formación continua para fomentar y propiciar una educación inclusiva.

Las TIC constituyen un gran apoyo en el aprendizaje de los alumnos con necesidades educativas, pues dan lugar a la utilización de metodologías nuevas y estrategias didácticas que facilitan la comunicación e interacción entre los discentes, tengan o no necesidades educativas (Martínez, Raposo, \& Añel, 2007; Córdoba, Cabero \& Soto, 2012; Zubillaga \& Alba, 2013; Troncoso, Martínez, \& Raposo, 2013; Pegalajar \& Colmenero, 2014). Otros autores nos dicen que el impulso que se les da a las TIC da lugar a una igualdad de oportunidades a los alumnos (Tedesco, 2006; Barroso \& Cabero, 2013; Marín, 2013). Así, autores como García García \& López Azuaga (2012) respaldan que las TIC pueden sustentar la base para el desarrollo de una educación para todos y todas.

Según Adell (2007), la integración de las TIC en las aulas da lugar a funciones distintas como son el acceso, como aprendizaje para usar adecuadamente la tecnología; la adopción, como apoyo a la forma tradicional de enseñanza y aprendizaje; la adaptación, como integración en las forma habitual de dar clase; la apropiación, como uso colaborativo, de proyectos y situaciones necesarias que se creen en el aula y la innovación, como descubrimiento a los nuevos usos de la tecnología y si combinación de diferentes modalidades.

Así, Cotrina García \& Cotrina García (2004 y 2007) sitúan a los usos integradores como herramientas de apoyo al aprendizaje y los usos inclusivos como un eje a la innovación. Los primeros centran sus finalidad en la compensación de las discrepancias desde el punto de vista individual. Por consiguiente la tecnología que se adapta y el software de apoyo que nos marcan el máximo exponente (Cabero, Córdoba \& Fernández Batanero, 2007). En el segundo de los casos, los usos inclusivos, nos da lugar a tener una educación con una progresión hacia la tolerancia, comunicación y relevancia en las aulas, 
al utilizar las TIC como herramienta de trabajo de forma individualizada o ajustada a las necesidades que cada alumno.

Todos los profesionales de la educación deben hacer una profunda reflexión de las funciones e importancia que realizan la TIC dentro del ámbito educativo actual y las oportunidades que ofrecen a las personas con discapacidad, de tal manera, que ayude a la toma de decisiones y contribuya a la creación de un sistema educativo de calidad para que el alumnado sea tratado de forma equivalente. Para dar este primer paso a la transformación e innovación educativa habrá que poner en movimiento mecanismos de concienciación a la utilización de las TIC y asimilar lo importante que es la formación continua junto a la investigación de este contenido.

Como se viene señalando por diferentes autores (García García \& López Azuaga, 2012; Marín, 2013; Cabero, Fernández Batanero \& Córdoba, 2016) las TIC abren un amplio abanico de posibilidades para poder conseguir paliar los defectos del sistema tradicional de enseñanza y proporcionar "entornos de aprendizaje con mayor potencial pedagógico", haciendo posible una igualdad de oportunidades entre todo el alumnado, tanto el que asiste de forma presencial en el aula como los que tienen que participar de forma virtual.

Autores como Doval (2011), expresan que sin la adecuada puesta en práctica de las TIC seguirá existiendo la exclusión social en el contexto educativo, con un aumento de la brecha digital entre el alumnado. Por lo que nos recomienda una oportuna valoración del acceso y competencia digital de los discentes, una introspección en su puesta en práctica y la determinación de la capacidad de las TIC.

Luque \& Rodríguez (2009, p. 6) proponen tener en cuenta una serie de pautas para futuras acciones:

- Integrar la tecnología en las aulas para alumnos con discapacidad

- Fomentar las ayudas para adquirir el equipamiento específico y los recursos necesarios por parte del centro

- Formación continua de los docentes en Software Educativo para alumnos con NEE

- Investigar sobre la metodología a utilizar con las TIC con alumnos con discapacidad

- Colaboración entre los docentes, la dirección del centro y los prodeuctores de software a través de redes regionales y nacionales

- Sensibilizar a la población sobre la importancia de una educación para todos y accesibilidad para la educación de las personas con NEE 
En nuestro entorno, a nivel general, los resultados de los estudios que se han realizado muestran cómo los docentes están capacitados para el uso de las TIC pero estos se sienten inseguros al tener que incorporarlas en los procesos de enseñanza-aprendizaje por no tener una buena perspectiva metodologógica (Fernández Batanero \& Bermejo, 2012; Prendes \& Gutiérrez, 2013; Suárez \& otros, 2013).

Como se ha visto a lo largo del documento y en varios estudios, las TIC nos ayudan a conseguir un proceso de enseñanza y aprendizaje de mayor calidad por lo tanto, debemos luchar por conseguir una mayor formación del profesorado en todas las etapas educativas para que sepan unar las TIC de manera adecuada con dichos alumnos. Debemos de tener en cuenta la gran diversidad presnete en nuestras aulas, todos los alumnos son diferentes y por ello, debemos de fomentar lo positivo de cada uno, luchando por una educación por y para todos. (Tello \& Cascales, 2015)

Tras analizar 49 publicaciones, las Tecnologías de la Información y de la Comunicación se revelan como una poderosa herramienta en la enseñanza del alumnado en situación de discapacidad y cuya aplicación en la práctica docente ofrece mayores oportunidades en el proceso de aprendizaje de los mismos.

Tal y como afirman Alba y Zubillaga (2013) y Colmenero y Pegalajar (2014), el uso de las Tecnologías de la Información y de la Comunicación, además de propiciar la comunicación y la interacción entre los individuos, favorecen diferentes estrategias metodológicas y didácticas promocionando el aprendizaje de alumnos y alumnas con necesidades educativas especiales.

Es necesario que los sistemas educativos se adapten a las individualidades de los alumnos y las alumnas. Atendiendo al preámbulo de la Ley Orgánica 2/2006, de Educación, modificada por la Ley Orgánica 8/2013, para la Mejora de la Calidad Educativa, hemos de considerar la educación como una herramienta para mejorar la condición humana y la vida colectiva.

Para la realización de la investigación que nos ocupa nos basamos en análisis de tipo documental, bibliográfico y de contenido.

Refiriéndonos específicamente al instrumento y análisis de datos, analizamos 49 publicaciones, recogiendo la información contenida en cada uno de ellos a través de fichas de registro en las que destacamos los siguientes datos: título, resumen, autoría, año de publicación, tipo de trabajo, TIC empleada, etapa educativa y palabras claves. A partir de ahí, siguiendo el modelo de Cabero, Fernández, y Córdoba (2016), realizamos una tabla de categorías (tabla 2) que nos permitiera analizar el contenido diferenciado. A pesar de que el DSM-V no lo considera un tipo de discapacidad, decidimos agregar a esta tabla de categoría el Trastorno del Espectro Autista, atendiendo a la cantidad de textos consultados que hacían referencia al mismo. 
Dadas las áreas de conocimiento contenidas en los 49 artículos revisados, concluímos que el acceso a las TIC (textos que se enfocan en recursos, dispositivos, etc. que ayudan a las personas con discapacidad y TEA está aumentando por beneficio de las TIC) es un área en la que se trabaja más.

Como afirman Garde, Giménez, Magal y Prefasi (2010) se está produciendo un cambio y cada vez son más los profesionales en educación sensibilizados con el uso e implementación de las TIC en las aulas y hacen una mayor demanda de dichas herramientas para superar la discriminación que este colectivo esta sufriendo.

Peñafiel (1999) señala una serie de principios en los que se ha de basar la utilización de las TIC en el ámbito educativo. De entre ellos destacamos la necesidad de ser conocedores de la influencia que tiene la incorporación de estas tecnologías en los procesos de transformación de la escuela en particular y la sociedad en general. Es por esto que deberemos considerar aspectos organizativos, metodologías, criterios de evaluación, etc., de manera que los recursos se adecúen a las singularidades de cada centro educativo y a las necesidades que presente su alumnado.

Cabe destacar que existen ciertos factores poco favorables en relación al uso de las TIC. Así, siguiendo a Cabero, Córdoba y Fernández (2007), destacamos los siguientes:

- el tipo y grado de las necesidades educativas especiales del alumnado y la discapacidad o trastorno del que se derivan.

- la necesidad de enfocar su utilización tanto desde el software como desde el software.

El problema más grande al que se enfrenta el profesorado a la hora de incorporar las TIC en el aula y ponerlas en práctica es la baja formación que reciben, tanto en conocimiento como práctica y dominio tecnológico. Este es un factor que incide directamente en las actitudes y el uso de las tecnologías que los docentes realizan. Por lo tanto, la formación recibida en la universidad se considera determinante en este aspecto. (Cabero-Almenara \& Guerra-Liaño, 2011; Molina-Jaén, PérezGarcía \& Antiñolo-Piñar, 2012; Ramírez-Orellana, Cañedo-Hernández \& Clemente-Linuesa, 2012).

Diferentes estudios acerca de la capacitación de los docentes para el manejo de las TIC coinciden en que las actitudes son favorables (Bullón, Cabero-Almenara, Llorente-Cejudo, Machuca-Portillo, Machuca-Portillo, Gallego-Pérez, Pérez-Diez de los Ríos \& PérezGarcía, 2008; Bustos-Navarrete, 2012; Cabero-Almenara, Llorente-Cejudo \& Puentes-Puente, 2010; Prendes-Espinosa \& Gutiérrez-Porlán, 2013; Suárez-Rodríguez, Almerich, Gargallo-López \& Aliaga, 2013). Sin embargo, ante su integración en el aula aparecen ciertas inseguridades relacionadas más con su aplicación didáctica y metodológica que con los awspectos tecnológicos en sí.

\section{ANÁLISIS DEL CUESTIONARIO}




\section{1. pregunta}

Sexo:

- hombre (azul) participantes: 12

- mujer (rojo) participantes: 69

Los datos indican que, la mayoría de los encuestados eran mujeres (85,2\%), sextuplicando casi al encuestado por hombres $(14,8 \%)$.

\section{Sexo}

81 respuestas

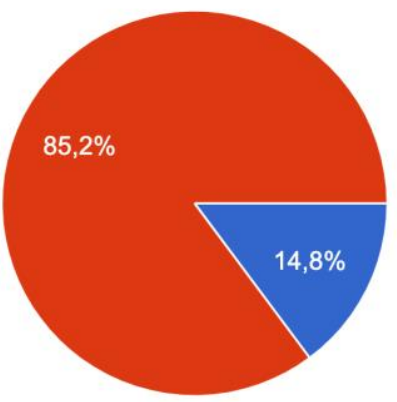

\section{2. pregunta}

El uso de las TIC con alumnos con NEE favorece su inclusión en el aula

$-1(1.2 \%)$ participantes 1

$-2(2,5 \%)$ participantes 2

\section{-3 (participantes 18)}

-4 (participantes 29)

-5 (participantes 31)

Los encuestados se sitúan en una horquilla amplia donde ven favorable el uso de las TIC con alumnos con NEE para el beneficio de la inclusión de estos, resultando un gran porcentaje en estar muy de acuerdo con esta afirmación (38,3\%) seguido de los que están algo de acuerdo (35,8\%). 
El uso de las TIC con alumnos con NEE favorece su inclusión en el aula 81 respuestas

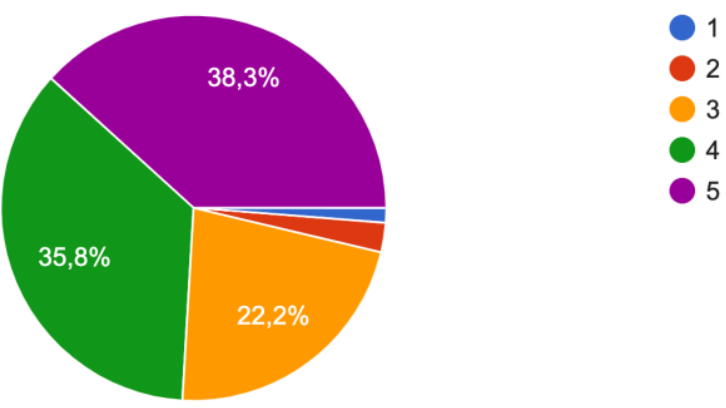




\section{3. pregunta}

Las TIC es un método que incrementa la motivación y refuerza la atención de los alumnos con NEE

\section{$-1(1.2 \%)$ participantes 1}

\section{-2 participantes 0}

\section{$-3(7,4 \%)($ participantes 6$)$}

\section{-4 (participantes 37)}

\section{-5 (participantes 37)}

Afirman los encuestados que las TIC es un método que motiva y refuerza la atención del alumnado con NEE, siendo un porcentaje muy alto el que está muy de acuerdo $(45,7 \%)$ y la misma cifra para los que comentan que están algo acuerdo con ello, frente al 7,4\% que nos dicen que está solo de acuerdo.

Las TIC es un método que incrementa la motivación y refuerza la atención de los alumnos con NEE

81 respuestas

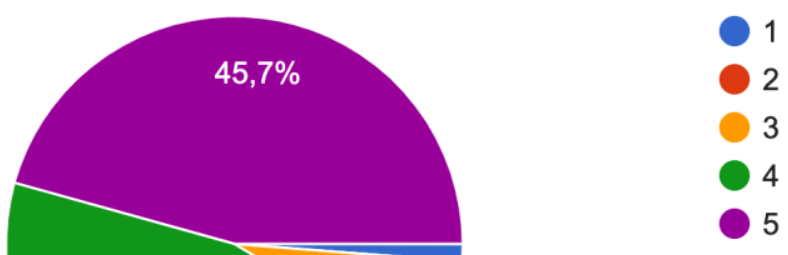

\section{4. pregunta}

Las TIC favorecen la autonomía de los estudiantes con NEE, ya que las herramientas se pueden adaptar a las necesidades de cada alumno/a de forma personalizada.

\section{$-1(1.2 \%)$ participantes 1}

\section{$-2(2,5 \%)$ participantes 2}

\section{$-3(7,4 \%)$ (participantes 12$)$}

\section{-4 (participantes 32)}




\section{-5 (participantes 34)}

Los datos recogidos en la encuesta apuntan a una mayoría de personas (42\%) que relacionan la autonomía de los estudiantes con NEE con ser herramientas que se adaptan a las necesidades de dichos discentes, muy por debajo de los que afirman que esto no es así (2,5\%) o los que nos comentan que están muy en desacuerdo con esta afirmación $(1,2 \%)$.

Las TIC favorecen la autonomía de los estudiantes con NEE, ya que las herramientas se pueden adaptar a las nec...de cada alumno/a de forma personalizada.

81 respuestas

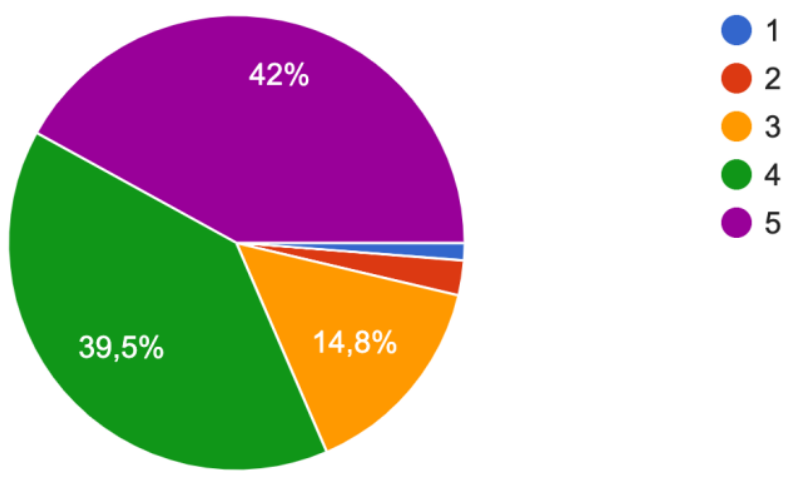

\section{5. pregunta}

El manejo de las herramientas TIC favorece al alumnado con NEE, haciendo que el profesorado ahorre tiempo y esfuerzo a estos si no saben manejar demasiado bien las herramientas TIC.

\section{-1 participantes 14}

\section{-2 participantes 9}

\section{-3 (participantes 25)}

-4 (participantes 17)

-5 (participantes 16)

Los datos señalan un reparto de cifras donde el manejo de las TIC como herramientas a la hora de ahorrar tiempo y esfuerzo al profesorado si no saben manejar estos instrumentos. Donde afirman estar 
muy de acuerdo el 19,8\%, algo de acuerdo el 21\%, de acuerdo el 30,9\%, algo en desacuerdo el 11,1\% y, por último, el 17,3\% muy en desacuerdo.

\section{El manejo de las herramientas TIC favorece al alumnado con NEE, haciendo que el profesorado ahorre tiempo y esfuerzo a es...ejar demasiado bien las herramientas TIC. 81 respuestas}

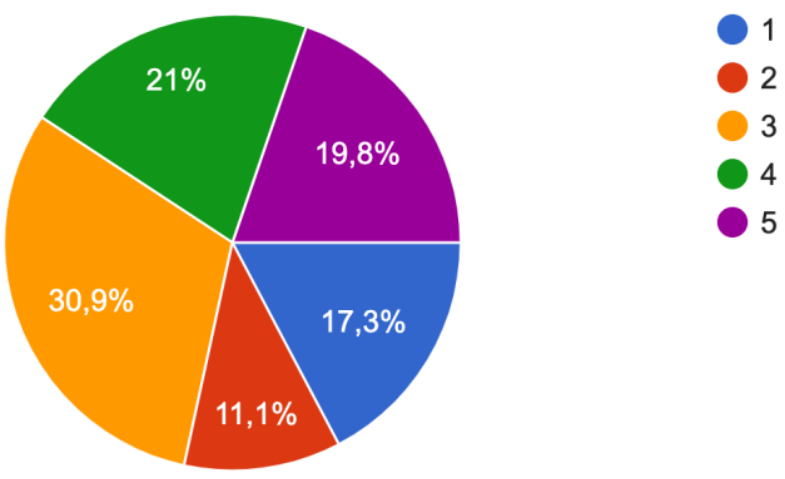

\section{6. pregunta}

La creatividad se ve favorecida con la utilización de las TIC ante las capacidades de autoaprendizaje de los niños con NEE.

\section{-1 participantes 4}

\section{-2 participantes 8}

\section{-3 (participantes 26)}

-4 (participantes 23)

\section{-5 (participantes 20)}

Como podemos observar, los datos de esta pregunta nos muestran como más del $50 \%$ de los encuestados están de acuerdo en afirmar que el uso de las TIC favorece la creatividad en los alumnos con NEE. Por otro lado, apenas el 15\% de los encuestados no creen que el uso de ordenadores o tablets, entre otros, puedan favorecer siendo 4 personas las que están totalmente en desacuerdo. 
La creatividad se ve favorecida con la utilización de las TIC ante las capacidades de autoaprendizaje de los niños con NEE.

81 respuestas

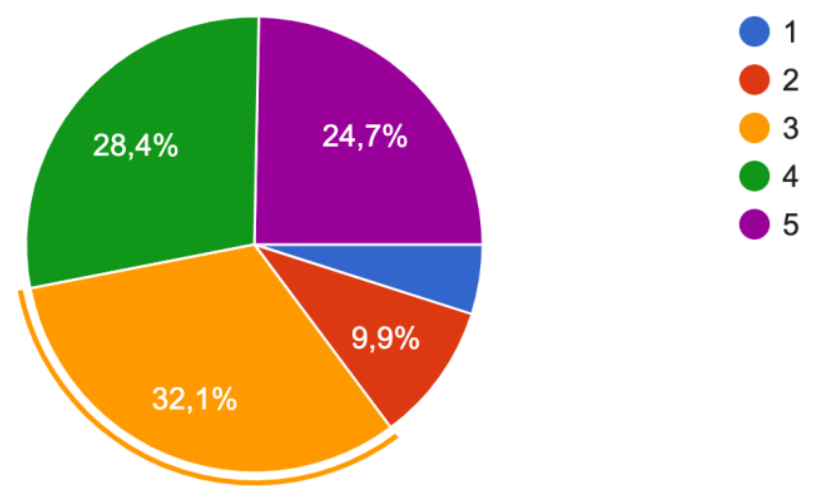

\section{7. pregunta}

Las TIC ofrecen la posibilidad de una evaluación dinámica continua y objetiva

\section{$-1(1,2 \%)$ participantes 1}

\section{$-2(7,4 \%)$ participantes 6}

\section{-3 (participantes 17)}

-4 (participantes 33)

\section{-5 (participantes 24)}

En el gráfico se puede apreciar cómo tan solo un $8 \%$ de los encuestados no están de acuerdo en que las TIC puedan ofrecerlo mientras que un gran porcentaje (más de la mitad) están de acuerdo en afirmar y reconocer la gran posibilidad que estas ofrecen siendo la evaluación más simple, dinámica y lúdica para los alumnos. 
Las TIC ofrecen la posibilidad de una evaluación dinámica continua y objetiva.

81 respuestas

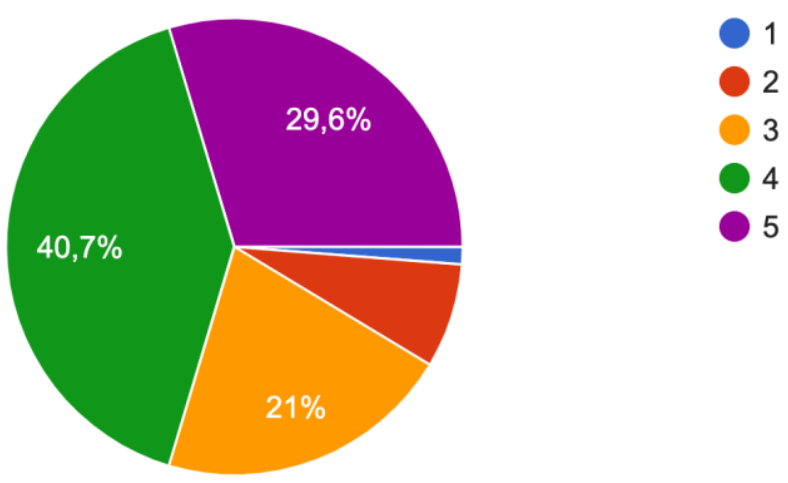

\section{8. pregunta}

Las TIC ayudan a superar las limitaciones que se derivan de las discapacidades cognitivas, sensoriales y motóricas del alumnado con NEE frente al resto de sus compañeros.

\section{$-1(3,7 \%)$ participantes 3}

\section{$-2(4,9 \%)$ participantes 4}

\section{-3 (participantes 22)}

-4 (participantes 26)

\section{-5 (participantes 26)}

En este ítem, echando un vistazo a la gráfica observamos que casi el 100\% de los encuestados (el 91,9\%) están de acuerdo en afirmar las posibilidades que le ofrecen las TIC a los alumnos ayudándoles a superar sus barreras y tener una educación de calidad. Sin embargo, hay un $9 \%$ que se muestra en desacuerdo totalmente. 
Las TIC ayudan a superar las limitaciones que se derivan de las discapacidades cognitivas, sensoriales y motóricas del al...con NEE frente al resto de sus compañeros. 81 respuestas

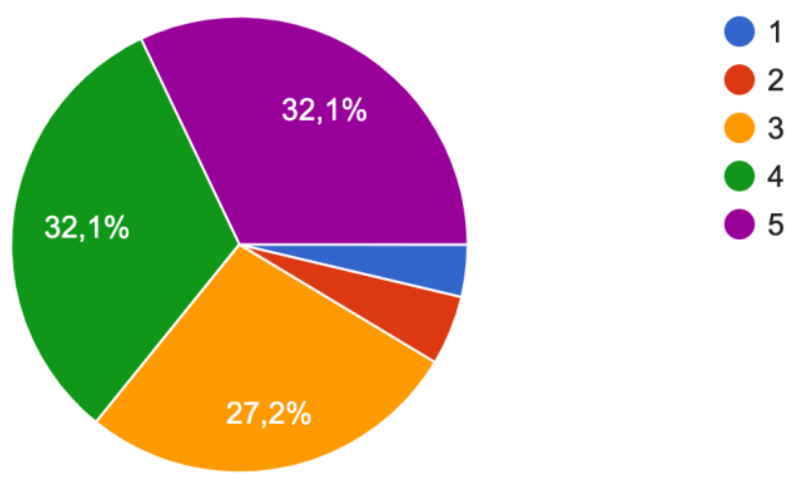

\section{9. pregunta}

Las TIC facilitan la inserción sociolaboral de los alumnos con dificultades específicas al usarse desde el comienzo de su vida educativa.

\section{$-1(1,2 \%)$ participantes 1}

\section{$-2(2,5 \%)$ participantes 2}

\section{-3 (participantes 17)}

-4 (participantes 31)

-5 (participantes 30)

La mayoría de los encuestados afirman estar de muy de acuerdo (37\%) o algo de acuerdo (38,3\%) en que las TIC son herramientas que ayudan en la inserción laboral del alumnado con NEE, si estas comienzan a utilizarse desde el comienzo de la vida educativa de estos. Frente al $21 \%$ que nos indica estar solo de acuerdo con esta afirmación, al contrario que nos indican los encuestados con solo el 2,5\% o el $1,2 \%$ que indican estar en desacuerdo o muy en desacuerdo con dicha pregunta. 
Las TIC facilitan la inserción sociolaboral de los alumnos con dificultades específicas al usarse desde el comienzo de su vida educativa.

81 respuestas

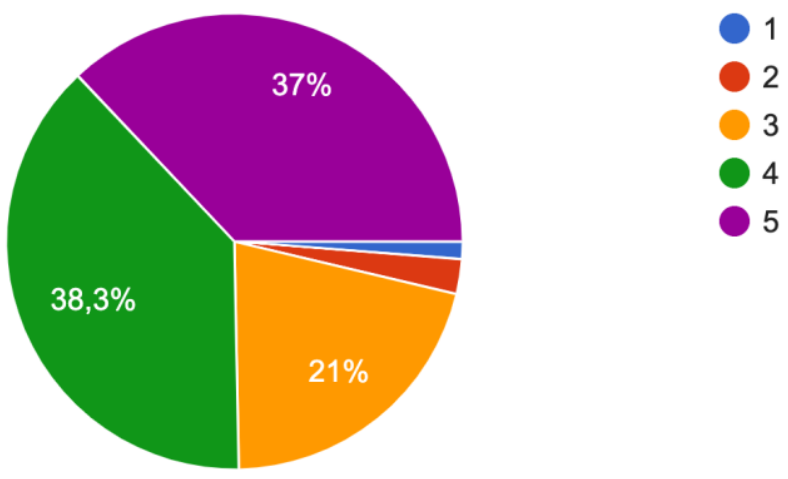

\section{0. pregunta}

Conociendo el valor que supone incluir las innovaciones tecnológicas en el proceso de transformación de la escuela y la sociedad, debemos adecuar los recursos disponibles a las necesidades de nuestros alumnos y a las características personales de la propia institución escolar, teniendo en cuenta los aspectos organizativos, metodologías, criterios de evaluación, etc

\section{$-1(1,2 \%)$ participantes 1}

\section{$-2(2,5 \%)$ participantes 2}

\section{-3 (participantes 10)}

\section{-4 (participantes 26)}

\section{-5 (participantes 42)}

Obtenemos un 51,9\% en la afirmación que nos dice que la inclusión de tecnologías en el proceso de transformación de la escuela y la sociedad deben adecuarse a los recursos disponibles de las necesidades de los alumnos y a las características personales de la propia institución, teniendo en cuenta los aspectos organizativos, metodológicos, metodologías, criterios de evaluación, etc. Seguido del 32,1\% que afirma estar algo de acuerdo junto al $12,3 \%$ que está de acuerdo. Por contra solo el 2,5\% y el 1,2\% aseguran estar en desacuerdo o muy en desacuerdo. 
Conociendo el valor que supone incluir las innovaciones tecnológicas en el proceso de transformación de la escuela y l...etodologías, criterios de evaluación, etc.

81 respuestas

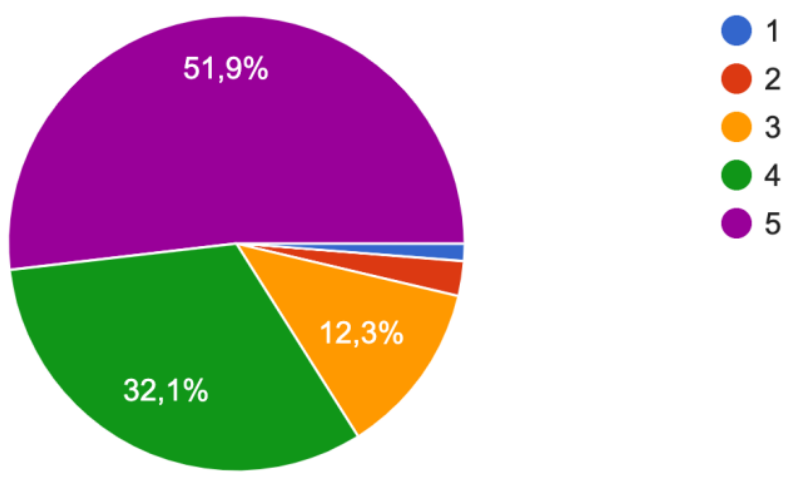

\section{1. pregunta}

La integración se ha preocupado por mejorar y cambiar la educación en vez de cambiar la cultura y la práctica tecnológica de las escuelas ordinarias.

\section{$-1(6,2 \%)$ participantes 5}

\section{$-2(4,9 \%)$ participantes 4}

\section{-3 (participantes 35)}

-4 (participantes 20)

-5 (participantes 17)

Más de la mitad de los encuestados (51,9\%) afirma estar totalmente de acuerdo con esta afirmación y el $32,1 \%$ se posiciona de acuerdo a la misma, considerando que el enfoque de la integración resulta insuficiente para atender las necesidades en materia tecnológica. Por el contrario, un 4,9\% de las personas consultadas está en desacuerdo y un 6,2\% totalmente en desacuerdo con la afirmación. 
La integración se ha preocupado por mejorar y cambiar la educación en vez de cambiar la cultura y la práctica tecnológica de las escuelas ordinarias.

81 respuestas

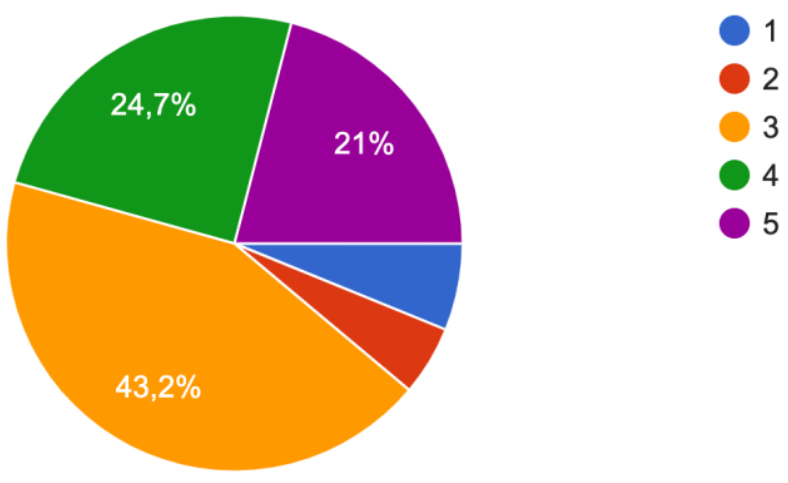

\section{2. pregunta}

La calidad de la educación que han recibido los alumnos con necesidades educativas especiales en el ámbito de la integración escolar ha sido escasa, puesto que se ha seguido manteniendo una dicotomía entre lo que hace el alumno con necesidades educativas especiales, de lo que hace el resto de sus compañeros en clase

\section{$-1(6,2 \%)$ participantes 5}

\section{$-2(4,9 \%)$ participantes 4}

\section{-3 (participantes 18)}

-4 (participantes 26)

-5 (participantes 28)

De entre los encuestados destaca un $21 \%$ se muestra totalmente de acuerdo con esta aseveración, y el $24,7 \%$ de acuerdo, frente a un $4,9 \%$ que dice estar en desacuerdo y un $6,2 \%$ totalmente en desacuerdo. 
La calidad de la educación que han recibido los alumnos con necesidades educativas especiales en el ámbito de la i... hace el resto de sus compañeros en clase. 81 respuestas

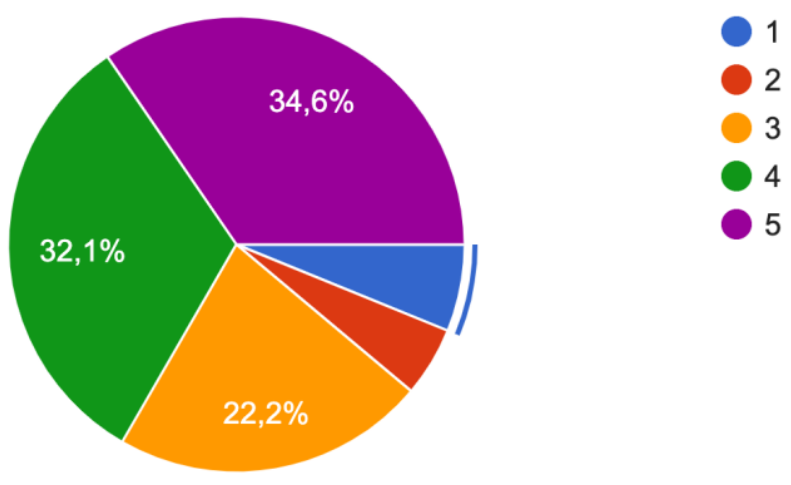

\section{3. pregunta}

Las necesidades específicas de algunos alumnos podrían verse compensadas solamente mediante la utilización de las TIC.

\section{-1 participantes 22}

\section{-2 participantes 17}

-3 (participantes 18)

-4 (participantes 15)

-5 (participantes 9)

Con respecto a este ítem, las respuestas han sido muy variadas ya que encontramos un gran porcentaje en todas ellas siendo minoritario "estoy totalmente de acuerdo". Así pues, encontramos un $27,2 \%$ en "totalmente en desacuerdo", un $21 \%$ en "poco de acuerdo" y un $22,2 \%$ entre el acuerdo y el desacuerdo. En esta pregunta podemos remarcar como la mayoría de personas no está de acuerdo con lo que dice el enunciado. 
Las necesidades especificas de algunos alumnos podrian verse compensadas solamente mediante la utilización de las TIC.

81 respuestas

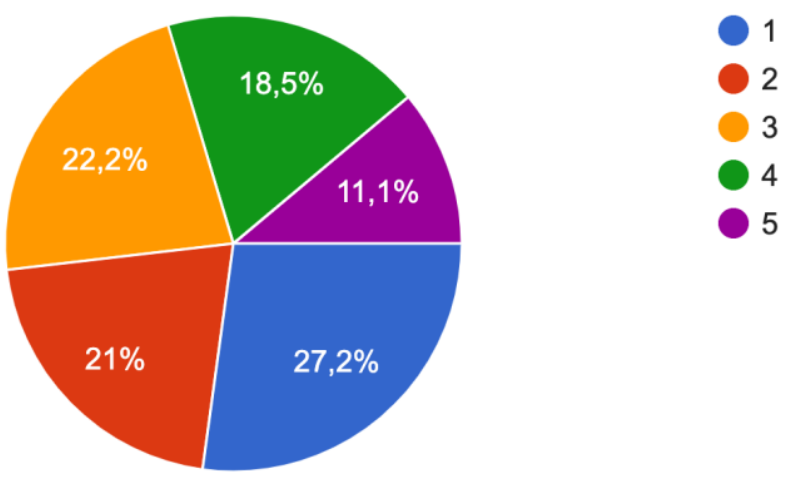

\section{4. pregunta}

Al rechazarse el uso de las herramientas TIC por parte del profesorado (Tecnofobia), se produce un beneficio en el alumnado con NEE al no usar en el aula las TIC

\section{-1 participantes 50}

-2 participantes 15

\section{-3 (participantes 12)}

$-4(3,7 \%)($ participantes 3$)$

\section{$-5(1,2 \%)($ participantes 1$)$}

Tal y como se desprende de la gráfica, la mayor parte de las personas encuestadas han mostrado encontrarse totalmente de acuerdo $(61,7 \%)$ con lo enunciado, mientras que solamente el 1,2\% afirma estar totalmente en desacuerdo, y un 3,7 en desacuerdo. 
Al rechazarse el uso de las herramientas TIC por parte del profesorado (Tecnofobia), se produce un beneficio en el...ado con NEE al no usar en el aula las TIC. 81 respuestas

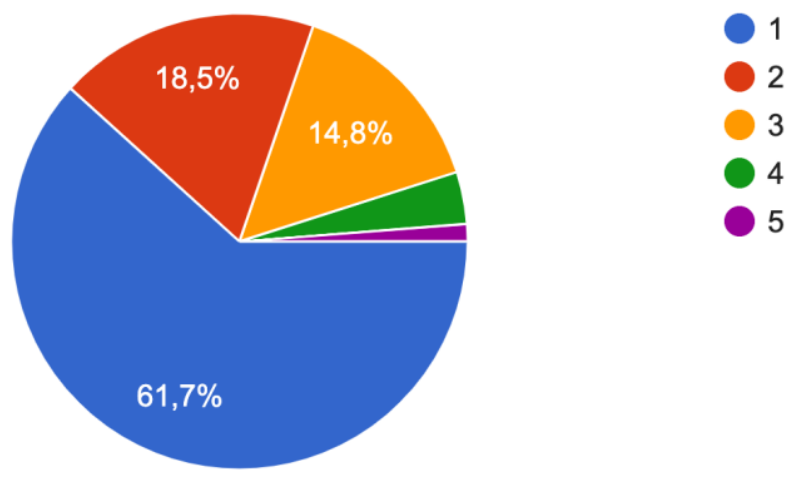

\section{5. pregunta}

La utilización de las TIC en el aula ordinaria dificulta la inclusión de los alumnos con NEE, puesto que su competencia digital es menor.

\section{-1 participantes 35}

\section{-2 participantes 18}

\section{-3 (participantes 17)}

\section{-4 (participantes 8)}

\section{$-5(3,7 \%)($ participantes 3$)$}

La utilización de las TIC en el aula ordinaria dificulta la inclusión de los alumnos con NEE, puesto que su competencia digital es menor.

81 respuestas

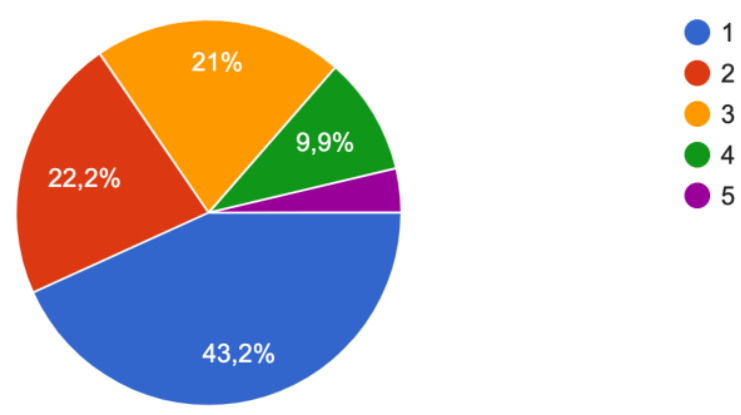


Los resultados de la encuesta muestran una mayoría de encuestados que dice estar totalmente en desacuerdo $(43,2 \%)$ o en desacuerdo $(22,2 \%)$ con el enunciado planteado, frente a una parte notablemente menor que se muestra de acuerdo $(9,9)$ o totalmente de acuerdo $(3,7 \%)$. Por tanto, es un número considerablemente mayor de docentes el que considera que la utilización de las TIC no supone dificultad alguna para la inclusión del alumnado con necesidades especiales.

\section{DISCUSIÓN/VALORACIÓN:}

El presente trabajo nos hace tener una visión global sobre que las TIC son necesarias para que los discentes con necesidades educativas especiales, puedan realmente tener una educación inclusiva, necesitando para ello el compromiso de todos los profesionales de la educación.

$2^{\mathrm{a}}$ pregunta

Esto se demuestra en la afirmación de Garde, Giménez, Magal y Prefasi (2010) donde nos comentan que los educadores cada día están más concienciados y se implican en la realización de nuevas herramientas, para que este colectivo de alumnos quede integrado en sus aulas, de ello se desprende el amplio abanico que suman los maestros que están muy de acuerdo y algo de acuerdo con un 74,1\% en nuestra encuesta.

\section{$3^{\mathrm{a}}$ pregunta}

De nuestra encuesta se desprende que las TIC son un método que motiva y refuerza la atención del alumnado con NEE, tal y como afirma Román, Cardemil y Carrasco (2011). Así nuestros encuestados afirman mayoritariamente con un 45,7\% esta afirmación frente al 1,2\% que asegura no estar nada de acuerdo.

$4^{\mathrm{a}}$ pregunta

Según Cabero, Córdoba y Fernández (2007) cuando analizan las ventajas del uso de las TIC sostienen que favorecen la autonomía del alumnado con NEE, al igual que la afirmación que extraemos al analizar los resultados de la encuesta realizada donde $42 \%$ afirman están muy de acuerdo y el 39,5\% afirma estar algo de acuerdo, en que las TIC son herramientas que se pueden adaptar a cada necesidad.

\section{$5^{\mathrm{a}}$ pregunta}

Autores como López y López (1995) en Paula (2003) nos dicen que es un inconveniente la falta de preparación de los educadores si estos no saben manejar las herramientas TIC, afirmación que queda 
confirmada en el reparto de cifras que nos da la respuesta, a nuestra pregunta en la encuesta, donde las cifras nos muestran un $19,8 \%$, un $21 \%$ y un 30,9\%, dando así un amplio abanico de quien aseguran estar muy de acuerdo, algo de acuerdo o de acuerdo, con ello. Frente al 11,1\% o 17,3\% que afirman están en desacuerdo o muy en desacuerdo.

\section{$6^{\mathrm{a}}$ pregunta}

Según Torres (1990), el uso de ordenadores con niños con NEE ofrece muchas ventajas entre las cuales destacamos la estimulación de la creatividad. Como podemos observar, los datos de esta pregunta nos muestran como más del 50\% de los encuestados están de acuerdo en afirmar que el uso de las TIC favorece la creatividad en los alumnos con NEE. Por otro lado, apenas el 15\% de los encuestados no creen que el uso de ordenadores o tablets, entre otros, puedan favorecerla.

\section{$7^{\mathrm{a}}$ pregunta}

En la misma línea que la pregunta anterior, según Torres (1990), las TIC también ofrecen la posibilidad de realizar una evaluación continua a los alumnos y mucho más objetiva de modo que eliminamos la subjetividad que pudiese aparecer en esta parte. En el gráfico se puede apreciar cómo tan solo un $8 \%$ de los encuestados no están de acuerdo en que las TIC puedan ofrecerlo mientras que un gran porcentaje (más de la mitad) están de acuerdo en afirmar la gran posibilidad que estas ofrecen siendo la evaluación más simple, dinámica y lúdica para los alumnos.

$8^{\mathrm{a}}$ pregunta

Una de las ventajas que algunos autores como Cabero, Córdoba y Fernández (2007) destacan con respecto al uso de las TIC es la posibilidad que ofrecen a los alumnos con NEE. Esta consiste en ayudar a superar las limitaciones que se derivan de sus discapacidades.

En este ítem, echando un vistazo a la gráfica observamos que casi el 100\% de los encuestados (el 91,9\%) están de acuerdo con dichos autores en afirmar las posibilidades que le ofrecen las TIC a dichos alumnos ayudándoles a superar sus barreras y tener una educación de calidad.

$9^{\text {a }}$ pregunta

Autores como Cabero, Córdoba y Fernández (2007) atribuyen una serie de ventajas al uso de las TIC para estudiantes con NEE. Entre estas destacamos la que el uso de las TIC facilita la inserción sociolaboral de alumnos/as con dificultades específicas. Así pues, echando un vistazo al gráfico podemos observar como la gran mayoría de los encuestados está de acuerdo con dichos autores. 
$10^{\mathrm{a}}$ pregunta

A lo largo del informe, hemos podido comprobar como hay un gran número de personas que defiende el uso de las TIC con alumnos con NEE ya que supone muchas ventajas con respecto su proceso de enseñanza-aprendizaje. Además, para una educación de calidad se requiere el compromiso de todos los miembros de la comunidad educativa y adaptarse a las características de los alumnos. Echando un vistazo al gráfico podemos observar que más de tres cuartas partes de los encuestados afirma estar de acuerdo con lo expuesto.

$11^{\mathrm{a}}$ pregunta

A la vista de los resultados, podemos afirmar que una mayoría de las personas consultadas coinciden con López Melero $(1997,182)$ cuando afirma que la integración (...) ha tendido a mejorar a la Educación Especial pero no a la Educación General. (...) la preocupación central de la inclusión es transformar la cultura, la organización y las prácticas educativas de las escuelas comunes para atender a la diversidad de necesidades educativas de todo el alumnado.

$12^{\mathrm{a}}$ pregunta

La opinión mayoritaria se corresponde con lo expuesto por López Melero (2008, 25 y ss.) que destaca de entre las barreras didácticas existentes para una mayor incorporación de las TIC en nuestro sistema educativo: El currículum basado en disciplinas y en el libro de texto en vez de partir de una concepción amplia del currículum (basado en situaciones problemáticas), teniendo en cuenta que la diversidad es la norma. (...)

\section{$13^{\mathrm{a}}$ pregunta}

La opinión predominante de entre los encuestados, a lo largo del cuestionario, es que la utilización de las TIC como herramienta para atender a la diversidad del alumnado presenta diversas y variadas ventajas. Sin embargo, no se considera que este uso sea suficiente para compensar las necesidades específicas del alumnado.

$14^{\mathrm{a}}$ pregunta

Gran parte de los docentes encuestados opinan que, como apunta López y López (1995) entre uno de los principales inconvenientes en el uso de las TIC encontramos: "La falta de preparación de los profesionales de la educación, produciéndose en algunos casos incluso rechazo por el uso de estas herramientas (tecnofobia)". 


\section{$15^{\mathrm{a}}$ pregunta}

Respecto a este ítem, la mayoría de los consultados parece posicionarse del lado de autores como Cabero, Córdoba y Fernández (2007), que atribuyen como una de las ventajas al uso de las TIC para estudiantes con NEE., el hecho de que "Ayudan a superar las limitaciones que se derivan de las discapacidades cognitivas, sensoriales y motóricas del alumnado.” 


\section{BIBLIOGRAFÍA}

Adell, J. (2007). Internet en el aula: las WebQuest. En J. Cabero \& J. Barroso (Eds.), (pp.211-225). Granada: Editorial Octaedro Andalucía.

Barroso, J. \& J. Cabero (2013), Nuevos escenarios digitales, Madrid, Pirámide.

Blanco, R. (2008). Marco conceptual sobre educación inclusiva. 48a Reunión de la Conferencia Internacional de Educación. La educación inclusiva: el camino hacia el futuro. Documento Final. Recuperado de:

http://www.uam.es/personal_pdi/stmaria/sarrio/declaraciones\%20manifiestos/ICE_FINAL_REPORT_s pa\%5B1\%5D.pdf.

Bullón, P.; Cabero-Almenara, J.; Llorente-Cejudo, M.C.; Machuca-Portillo, M. C.; Machuca-Portillo, G.; Gallego-Pérez, Ó.; Pérez-Diez de los Ríos, J. L. \& Pérez-García, R. (2008). Alfabetización digital de los estudiantes de la Facultad de Odontología de la Universidad de Sevilla. Sevilla: Grupo de Investigación Didáctica, GID, Universidad de Sevilla. Disponible en: https://idus.us.es/xmlui/handle/11441/32265

Bustos-Navarrete, C. (2012). Creencias docentes y uso de nuevas tecnologías de la información y comunicación en profesores de cinco establecimientos chilenos de educación básica y media. Universitas Psychologica, 11 (2), 511-521. Disponible http://revistas.javeriana.edu.co/index.php/revPsycho/article/download/3042/2234

Cabero, J. Fernández Batanero, J. M. \& Córdoba, M. (2016). Conocimiento de las TIC Aplicadas a las Personas con Discapacidades. Construcción de un Instrumento de Diagnóstico. En: Magis, Revista Internacional de Investigación en Educación, Vol. 8. Núm. 17, 157-176.

Cabero, J., Córdoba, M. y Fernández Batanero, J.M. (2007). Las TIC para la igualdad. Sevilla: Eduforma.

Cabero, J. \& Guerra, S. (2011). La alfabetización y formación en medios de comunicación en la formación inicial del profesorado. Educación XX1, 14, 1, 89-115.

Córdoba, M. Cabero, J. \& Soto, F.J. (2012). Buenas prácticas de aplicación de las TIC para la igualdad. Sevilla: Eduforma.

Cotrina García, M. \& García García, M. (2007). Las TIC como herramientas facilitadoras de la Atención a la Diversidad. IPLAND (et al). La atención a la Diversidad: una responsabilidad compartida. Huelva: Servicio de Publicaciones de la Universidad de Huelva.

Doval, M. (2011). Tecnologías de apoyo a la diversidad en la escuela inclusiva. En M. Cebrián \& M. Gallego (Eds.), Procesos educativos con TIC en la sociedad del conocimiento (45-57). Madrid: Pirámide.

Escudero, J. M. \& Domínguez, B. (2012). Las políticas de lucha contra el fracaso escolar: ¿programas especiales o cambios profundos del sistema y la educación? Revista de Educación, no extraordinario, 174193.

Fernández Batanero, J. M. y Bermejo, B. (2012). Actitudes docentes hacia las TIC en centros de buenas prácticas educativas con orientación inclusiva, en Enseñanza \& Teaching, 30(1), pp. 45-61.

García García, M. \& López Azuaga,R. (2012). Explorando desde una perspectiva 
inclusiva el uso de las TIC para atender a la diversidad. Profesorado. Revista de curriculum y formación del profesorado, 16 (1). Recuperado de http://www.ugr.es/local/recfpro/rev161COL4.pdf.

Luque, D., \& Rodríguez, G. (2009). Tecnología de la Información y Comunicación aplicada al alumnado con discapacidad: un acercamiento docente, en Revista Iberoamericana de Educación, 49(3), pp. 1-8. Recuperado de https://goo.gl/Hw8hxg

Marín, V. (coord.) (2013), Desarrollando la competencia digital desde la educación inclusiva, Mataró, Da Vinci.

Martínez, E., Raposo, M. \& Añel, E. (2007). La potencialidad de los materiales en la promoción de la escuela inclusiva. Revista Iberoamericana de Evaluación Educativa, 5, 3, 47-63.

Molina et al. (2012). Las TIC en la formación inicial y en la formación permanente del profesorado de infantil y primaria. EDUTEC, Revista Electrónica de Tecnología Educativa, 41. Recuperado de http://edutec.rediris.es/Revelec2/Revelec41/TIC

formacion_inicial_permanente_profesorado_infantil_primaria.html

Pegalajar, M.C. \& Colmenero, M.J. (2014). Estudio piloto sobre el uso de las redes sociales en jóvenes con discapacidad intelectual. EDUTEC, Revista Electrónica de Tecnología Educativa, 48. Recuperado de http://edutec.rediris.es/Revelec2/Revelec48/n48_Pegalajar_Colmenero.html.

Peñafiel, F. (1999): Los recursos informáticos y la escolarización de alumnos con necesidades educativas. En A. Sánchez y otros: Los desafíos de la Educación Especial en el umbral del siglo XXI. Actas de las XVI Jornadas Nacionales de Universidad y Educación Especial. Almería.

Prefasi, S., Magal, T., Garde, F. y Giménez, J.L (2010). Tecnologías de la 64 Información y de la Comunicación orientadas a la educación de personas con discapacidad cognitiva, Revista Latinoamericana de Tecnología Educativa.- RELATEC, 9(2), 107-123.

Prendes, M.P. \& Gutiérrez, I. (2013). Competencias tecnológicas del profesorado en las universidades españolas, en Revista de Educación, 361, pp.196-222.

Ramírez, E., Cañedo, I. \& Clemente, M. (2012). Las actitudes y creencias de los profesores de secundaria sobre el uso de Internet en sus clases. Comunicar, 38, 147-155.

Suárez, J. M., Almerich, G., Gargallo, B., y Aliaga, F.M. (2013). Las competencias del profesorado en TIC: estructura básica. Educación XX1, 16(1), 39-62. doi: 10.5944/educxx1.16.1.716

Tedesco, J.C. (2006), TIC y la desigualdad educativa en América Latina, en Ministerio de Educación de la República de Chile (ed.), Seminario "Las Tecnologías de Información y Comunicación y los Desafíos del Aprendizaje en la Sociedad del Conocimiento”, Santiago de Chile, Ministerio de Educación, pp. 101115.

Tello, I. \& Cascales, A. (2015). Las TIC y las necesidades específicas de apoyo educativo: análisis de las competencias TIC en los docentes, en RIED, 16(2), pp. 355-383. 
Troncoso, A.B., Martínez, M.E. y Raposo, M. (2013). La inclusión del alumno con discapacidad intelectual a partir del uso de blogs: una experiencia educativa innovadora. Revista Latinoamericana de Inclusión Educativa, 7(2), 195-211. Recuperado de http://www.rinace.net/rlei/numeros/vol7-num2/art11.pdf.

Zubillaga, A. \& Alba, C. (2013). La discapacidad en la percepción de la tecnología entre estudiantes universitarios. Comunicar, 40 (XX), 165-172. 